\title{
Taxonomic notes on some ant-mimicking jumping spiders (Araneae: Salticidae) from India
}

\author{
Таксономические заметки о некоторых муравьевидных \\ пауках-скакунчиках (Araneae: Salticidae) из Индии
}

\author{
John T.D. Caleb \\ Аж.Т.А. Камеб \\ National Centre for Biological Sciences, Tata Institute of Fundamental Research, Bangalore 560 065, Karnataka, India. \\ E-mail: caleb87woodgate@gmail.com
}

KEY WORDS: Ant-like jumping spiders, Myrmarachne, Salticidae, India, redescriptions.

КЛЮЧЕВЫЕ СЛОВА: муравьевидные пауки-скакунчики, Myrmarachne, Salticidae, Индия, переописания.

ABSTRACT. Five species of ant-mimicking jumping spiders are recorded from India: Myrmarachne kuwagata Yaginuma, 1967, M. melanocephala MacLeay, 1839, M. plataleoides (O. Pickard-Cambridge, 1869), M. prava (Karsch, 1880), and M. ramunni Narayan, 1915. All these species are redescribed and illustrated in detail.

РЕЗЮМЕ. Пять видов муравьевидных пауковскакунчиков отмечены из Индии: Myrmarachne kuwagata Yaginuma, 1967, M. melanocephala MacLeay, 1839, M. plataleoides (O. PickardCambridge, 1869), M. prava (Karsch, 1880), и $M$. ramunni Narayan, 1915. Все виды переописаны и детально иллюстрированы.

\section{Introduction}

The genus Myrmarachne MacLeay, 1839 (s.lat.) is characterized by its members exhibiting the Batesian mimicry towards ants [Wanless, 1978; Yamasaki, Edwards, 2013; Yamasaki, Ahmad, 2013]. The genus consists of 229 described species [WSC, 2016]. Based on the similarity of copulatory organs in some species groups of Myrmarachne (s.lat.), Prószyński [2016b] have erected nine new genera, to which 34 Myrmarachne species have then been transferred. Of them, 24 species of $M y r-$ marachne (s.str.) and one of Myrmaplata Prószyński, 2016 have been recorded from India to date [WSC, 2016; Prószyński, 2016b]. The members of Myrmarachne reported from India are poorly described and in most cases are unrecognizable by the existing original descriptions and illustrations [see Prószyński, 1992].

This paper is devoted to detailed morphological descriptions of the five Myrmarachne species collected from south India, namely: M. kuwagata Yaginuma, 1967, M. melanocephala MacLeay, 1839, M. plataleoides (O. Pickard-Cambridge, 1869), M. prava (Karsch, 1880) and M. ramunni Narayan, 1915.
Materials and methods

Spiders were collected from Araabath Lake and the campus of Madras Christian College in the vicinities of Chennai City (erstwhile Madras), Tamil Nadu, and from the campus of the National Centre for Biological Sciences, Bangalore, Karnataka. The specimens were photographed by means of DSLR Nikon D60, 18-55 mm (for macro capability, the lens was reversed and mounted on the camera body with help of an attachment ring). The specimens were preserved in $70 \%$ alcohol and examined by means of Leica S8APO stereoscopic microscope; photographs were obtained by means of Leica MC120 HD camera, with the LAS core software. Male palps were detached and studied. Female copulatory organs were removed by using a fine surgical scalpel. The epigynes were cleared in a $10 \% \mathrm{KOH}$ solution. The studied specimens have been deposited in the collection of the National Centre for Biological Sciences (NCBS), Bangalore. Descriptions are based on fresh specimens. All measurements are in $\mathrm{mm}$. The format of descriptions, measurements and leg spination follow Yamasaki [2010] and Yamasaki, Ahmad [2013]. Abbreviations used in the text are as follows: ALE - anterior lateral eye, AME - anterior median eye, di — distal, md - middorsal, PLE - posterior lateral eye, PME - posterior median eye, $\mathrm{pd}$ - prodorsal, $\mathrm{pr}$ - proximal, $\mathrm{pv}$ proventral, RTA — retrolateral tibial apophysis, rv retroventral.

\section{Taxonomy}

Myrmarachne MacLeay, 1839

Type species: Myrmarachne melanocephala MacLeay, 1839 


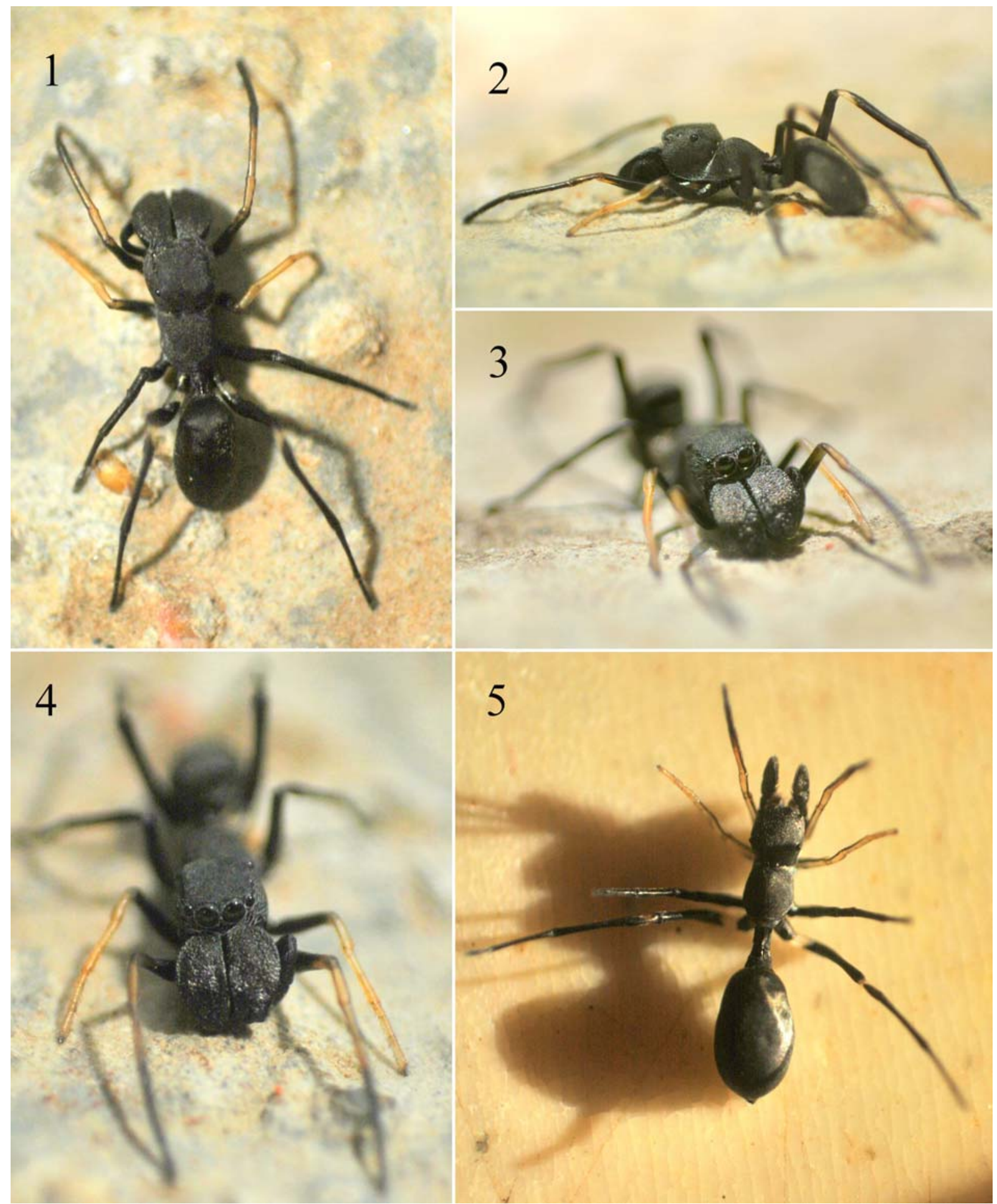

Figs 1-5. Myrmarachne kuwagata Yaginuma, 1967. 1 - male body, dorsal view; 2 - ditto, lateral view; 3, 4 - ditto, front view; 5 - female body, dorsal view.

Рис. 1-5. Myrmarachne kuwagata Yaginuma, 1967. 1 - тело самца, вид сверху; 2 - тоже, вид сбоку; 3, 4 - тоже, вид спереди; 5 - тело самки, вид сверху. 

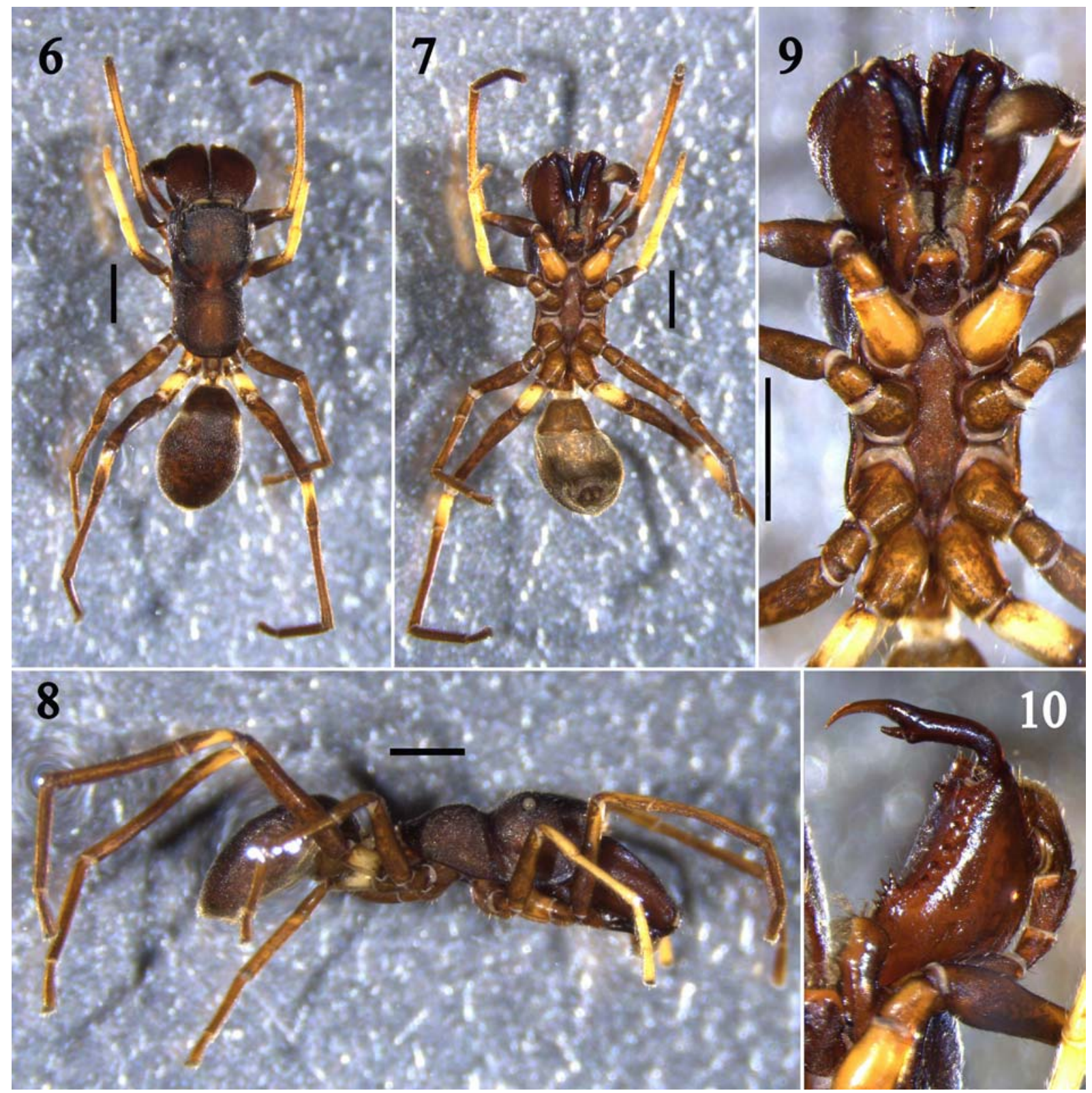

Figs 6-10. The male of Myrmarachne kuwagata Yaginuma, 1967. 6 - body, dorsal view; 7 - ditto, ventral view; 8 - ditto, lateral view; 9 - maxillae, labium, sternum and coxae; 10 - chelicerae, ventral view. Scale bars: $1 \mathrm{~mm}(6-9)$.

Рис. 6-10. Самец Myrmarachne kuwagata Yaginuma, 1967. 6 - тело, вид сверху; 7 - тоже, вид снизу; 8 - тоже, вид сбоку; 9 - максилла, лабиум, стернум и вертлуги; 10 - хелицеры, вид снизу. Масштаб: 1 мм (6-9).

Myrmarachne kuwagata Yaginuma, 1967 Figs 1-19, Map 1.

Myrmarachne kuwagata Yaginuma, 1967: 100, figs 31-p; Ono et al, 2009: 564, figs 37-39.

MATERIAL. INDIA: $10^{7}, 1$ (NCBS-AR127, AR128), scrub regions near Araabath Lake $\left(13.123335^{\circ} \mathrm{N}, 80.136958^{\circ} \mathrm{E}\right)$, Tamil Nadu, 21.73 m a.s.1., 18.08.2013, John T.D. Caleb.

DESCRIPTION. MALE. Total length 5.40; carapace 2.57 long, 1.41 wide; abdomen 2.35 long, 1.40 wide. Width of eye row I 1.19; II 1.17; III 1.27. Eye size: AME 0.39, ALE 0.16, PME 0.07, PLE 0.19;
ALE-PME 0.28, ALE-PLE 0.65. Chelicerae length 1.48. Sternum length 1.50. Leg spination; Patella I rv 1; tibia I pv 5, rv 5; metatarsus I pv 2, rv 2; tibia II pv 1, rv 3 ; metatarsus II pv 2, rv 2. Cephalic part flat dorsally, finely rugulose, covered with fine grey hairs (Figs 1, 6). Thoracic region clearly separated with deep furrow, lower than cephalic region, convex dorsally, gradually sloping posteriorly (Figs 2, 8). Chelicerae shorter than carapace, black, rugulose, flattened dorsally and curved outer edges (Figs 3,4); chelicerae dentition with 6 promarginal teeth (5 proximal and 1 distal) and 7 ret- 


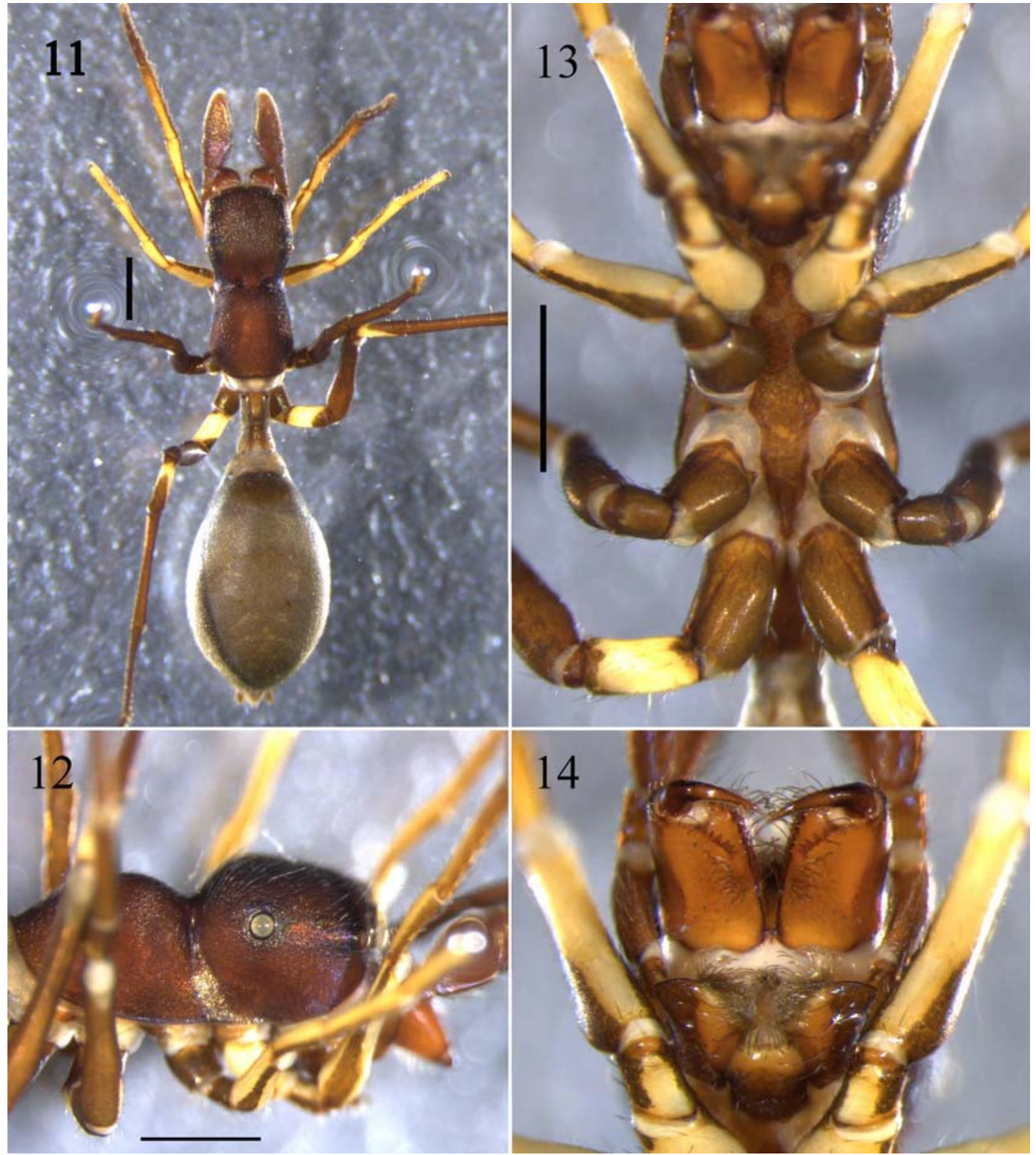

Figs 11-14. The female of Myrmarachne kuwagata Yaginuma, 1967. 11 - body, dorsal view; 12 - carapace, lateral view; 13 sternum and coxae; 14 - chelicerae, ventral view. Scale bars: $1 \mathrm{~mm}(11-13)$.

Рис. 11-14. Самка Myrmarachne kuwagata Yaginuma, 1967. 11 - тело, вид сверху; 12 - головогрудь, вид сбоку; 13 стернум и вертлуги; 14 - хелицеры, вид снизу. Масштаб: 1 мм (11-13).

romarginal teeth. Fang stout with a bump on the prolateral margin and a longer apophysis on the ventral region (Figs 10, 15). Sternum dark, slender, slightly overlapped by coxae I, II and III (Fig. 9). Legs dark brown, except for light yellow segments on the first two pairs of legs. Leg I with light yellow patella and tibia, leg II with lighter, yellowish patella, tibia, metatarsus and tarsus (Figs 6-8). Abdomen almost oval, covered with fine hairs (Fig. 1). Palps with rounded tegulum, with U-shaped seminal reservoir in the 12 to 1 o'clock position; embolus with two coils, RTA twisted, strongly curved, S-shaped and well developed flange (Figs 16, 17).
FEMALE. Total length 8.04; carapace 2.9 long, 1.35 wide; abdomen 4.00 long, 2.25 wide. Width of eye row I 1.23; II 1.22; III 1.38. Eye size: AME 0.38, ALE 0.13, PME 0.05, PLE 0.21; ALE-PME 0.31, ALE-PLE 0.72. Sternum length 1.64. Leg spination; Patella I rv 1; tibia I pv 5, rv 5; metatarsus I pv 2, rv 2; tibia II pv 3, rv 3; metatarsus II pv 2, rv 2. Coloration as in the male, but differs in having a deep, narrow constriction between cephalic and thoracic regions, thoracic region strongly convex dorsally (Figs 5, 11, 12). Chelicerae with 6 teeth on the prolateral margin and 7 teeth on the retrolateral margin (Fig. 14). Coxa I yellowish, coxa II-IV dark brown (Fig. 13). Thin dark brown stripes present on the 


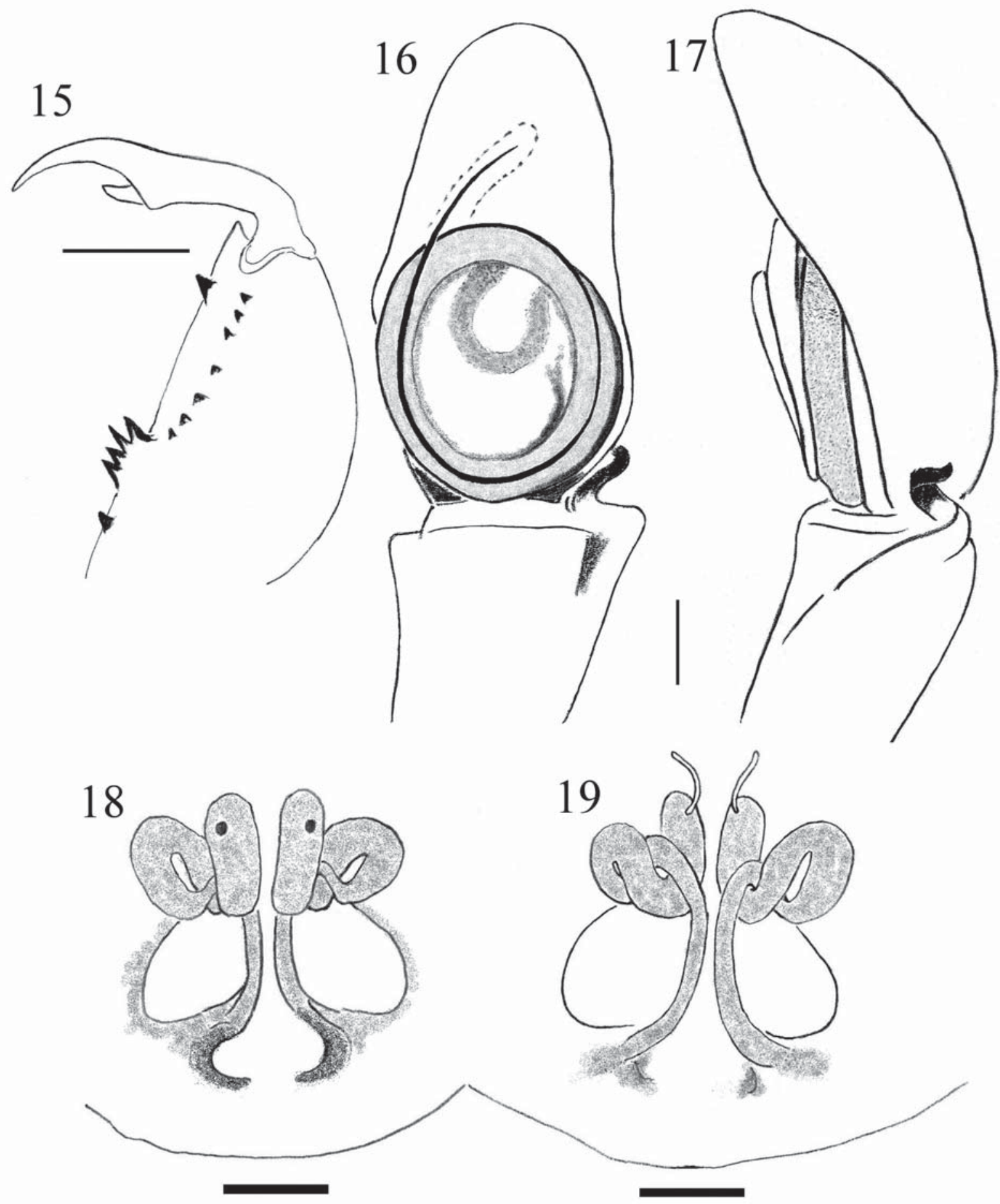

Figs 15-19. Chelicerae and copulatory organs of Myrmarachne kuwagata Yaginuma, 1967. 15 - chelicerae and fang, ventral view; 16 - palp, ventral view; 17 - ditto, retrolateral view; 18 - epigyne, ventral view; 19 - spermathecae, dorsal view. Scale bars: $0.5 \mathrm{~mm}$ (15), $0.1 \mathrm{~mm}(16-19)$.

Рис. 15-19. Хелицеры и копулятивные органы Myrmarachne kuwagata Yaginuma, 1967. 15 - хелицера, вид снизу; 16 пальпа, вид внизу; 17 - тоже, вид сбоку-сзади; 18 - эпигина, вид снизу; 19 - сперматека, вид сверху. Масштаб: 0,5 мм (15), 0,1 мм (16-19). 


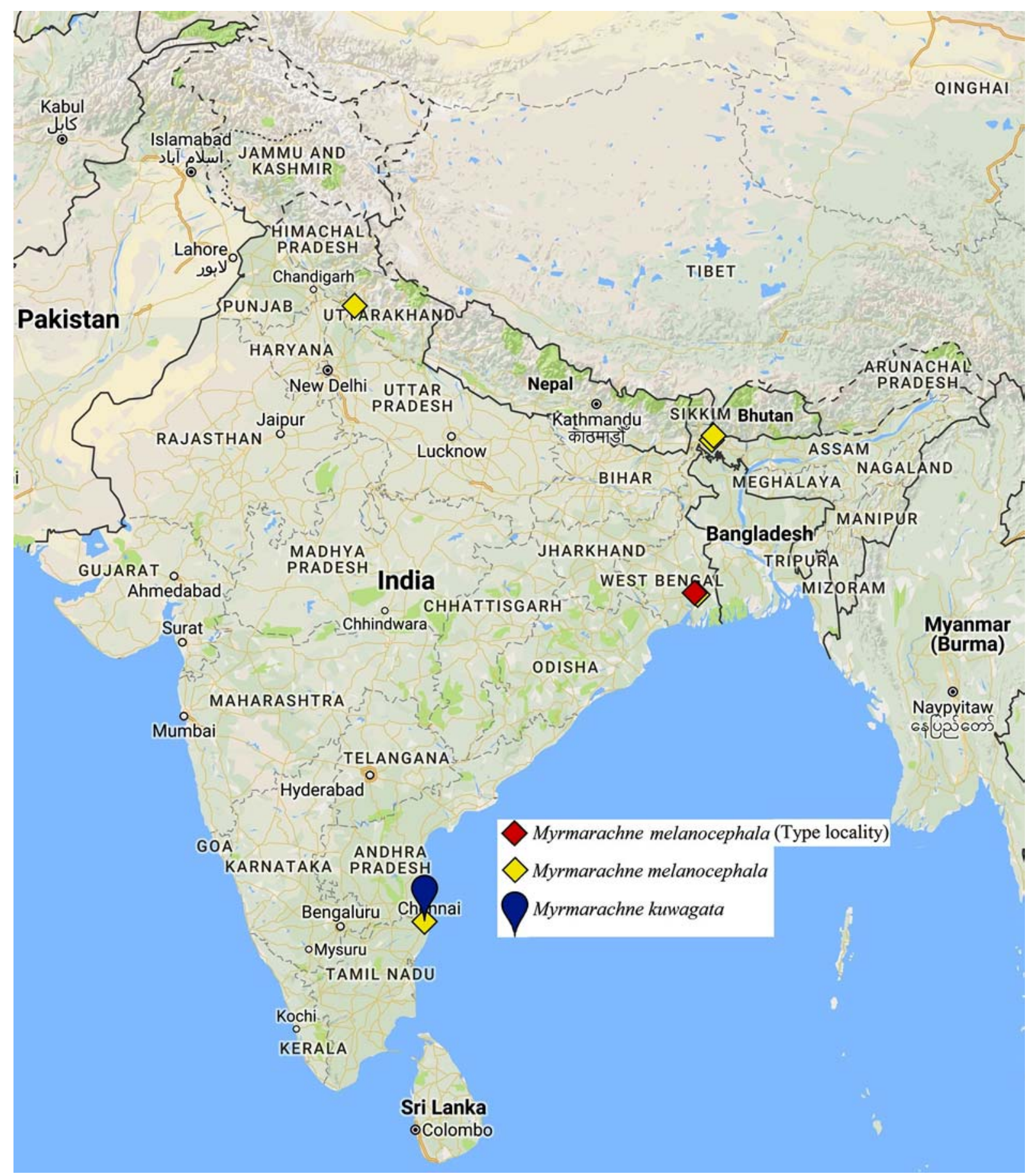

Map 1. Distributional records of Myrmarachne spp. in India. Карта 1. Точки находок Myrmarachne spp. в Индии.

lateral margins of first two pair of legs (Figs 11, 12). Epigyne with large, oval copulatory openings; sclerotized copulatory ducts thinner, forming twists before reaching spermathecae; lateral pockets present just above epigastric furrow (Figs 18, 19).

NATURAL HISTORY. Found among the scrub sites near Araabath Lake.
REMARKS. Considering the geographic gap between the type locality of this species and the Indian records (Map 1), the specimens from Tamil Nadu might belong to a different species. However, due to the lack of sufficient comparative data and specimens, the studied specimens have been provisionally assigned to $M$. kuwagata. 


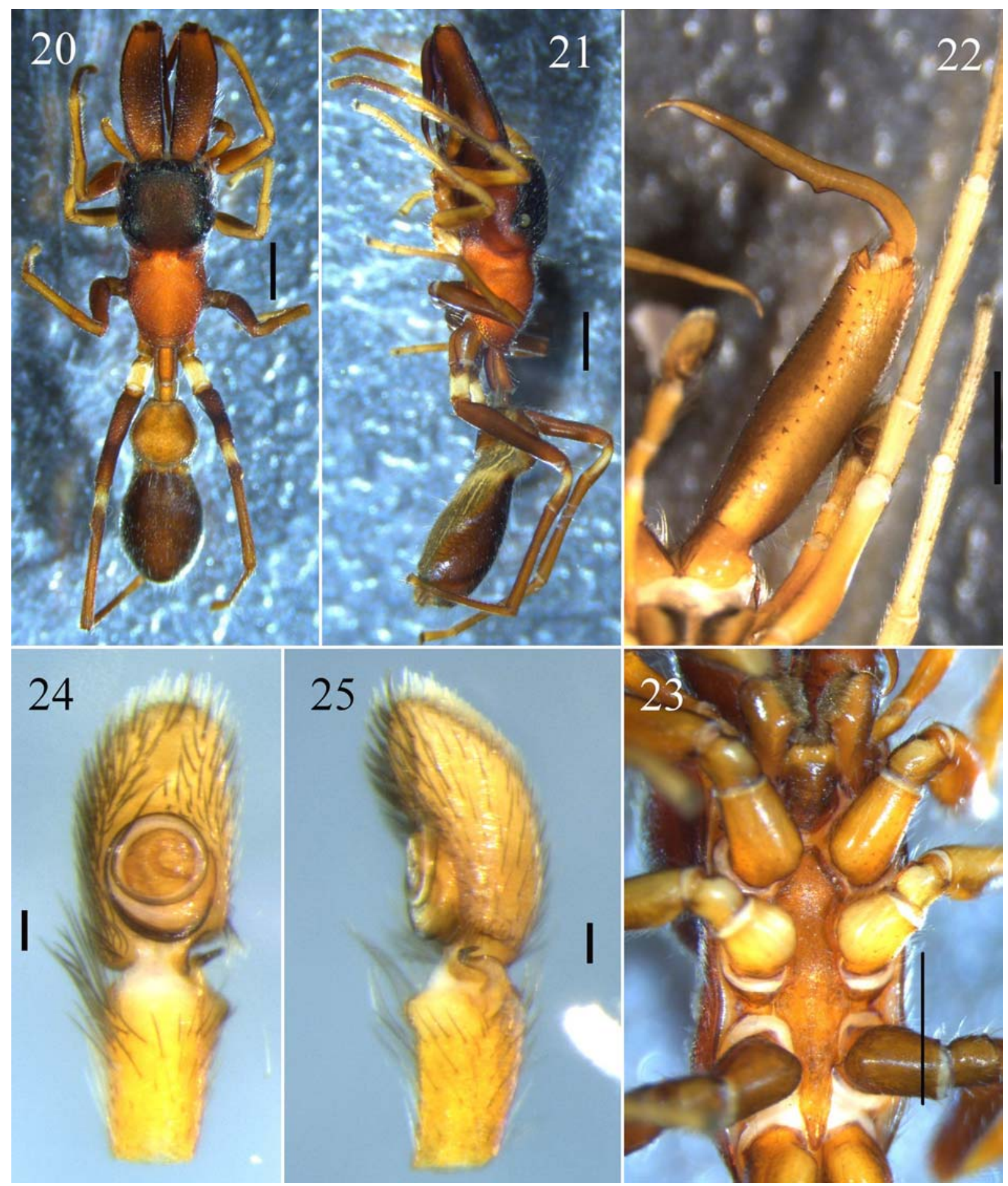

Figs 20-25. The male of Myrmarachne melanocephala MacLeay, 1839. 20 - body, dorsal view; 21 - ditto, lateral view; 22 chelicerae, ventral view; 23 - maxillae, labium, sternum and coxae; 24 - palp, ventral view; 25 - ditto, retrolateral view. Scale bars: 1 $\mathrm{mm}(20-23), 0.1 \mathrm{~mm}(24-25)$.

Рис. 20-25. Самец Myrmarachne melanocephala MacLeay, 1839. 20 - тело, вид сверху; 21 - тоже, вид сбоку; 22 - хелицеры, вид снизу; 23 - максилла, лабиум, стернум и вертлуги; 24 - пальпа, вид снизу; 25 - тоже, вид сбоку-сзади. Масштаб: 1 мм (2023), 0,1 мм (24-25). 

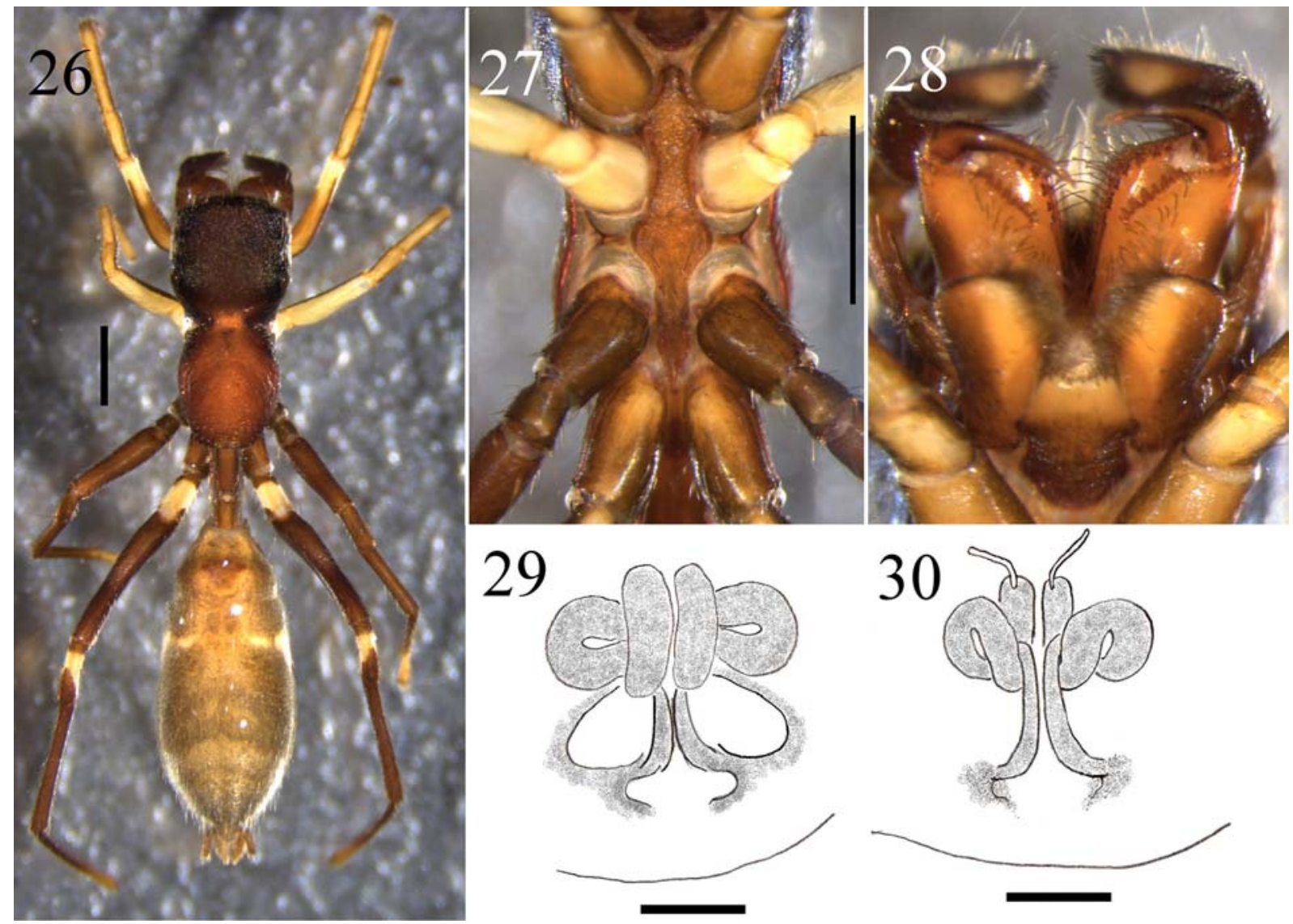

Figs 26-30. The female of Myrmarachne melanocephala MacLeay, 1839. 26 - body, dorsal view; 27 - sternum and coxae; 28 chelicerae and fang, ventral view; 29 - epigyne, ventral view; 30 - spermathecae, dorsal view. Scale bars: $1 \mathrm{~mm}(26,27) ; 0.1 \mathrm{~mm}(29$, 30).

Рис. 26-30. Самка Myrmarachne melanocephala MacLeay, 1839. 26 - тело, вид сверху; 27 - стернум и вертлуги; $28-$ хелицеры, вид снизу; 29 - эпигина, вид снизу; 30 - сперматека, вид сверху. Масштаб: 1 мм (26, 27); 0,1 мм (29, 30).

DISTRIBUTION. China, Korea, Japan [WSC, 2016], India (Chennai; Map 1).

\section{Myrmarachne melanocephala MacLeay, 1839 Figs 20-30, Map 1.}

Myrmarachne melanocephala MacLeay, 1839: 11, pl. 1, fig. 4; Edwards, Benjamin, 2009: 5, figs 1A-H, 2A-D, 3A-D, 4A-E, 5A-D; Yamasaki, Edwards, 2013: 15, figs 46-58; Yamasaki, Ahmad, 2013: 541, figs 32A-G, 33A-H, 34 A-C; Benjamin, 2015: 17, figs $17 \mathrm{~A}-\mathrm{D}, 18 \mathrm{~A}-\mathrm{D}, 19 \mathrm{~A}-\mathrm{D}$.

For a complete set of taxonomic references see WSC [2016].

MATERIAL. INDIA: $3 \sigma^{7} \sigma^{7}, 1$ (NCBS-AR129-AR132), Thirumullaivoyal $\left(13.125090^{\circ} \mathrm{N}, 80.135712^{\circ} \mathrm{E}\right), 26.85 \mathrm{~m}$ a.s.1., 18.08.2013, John T.D. Caleb; $1 \sigma^{7}, 1$ immature $q$ (author's personal collection), Thirumullaivoyal $\left(13.126197^{\circ} \mathrm{N}, 80.135966^{\circ} \mathrm{E}\right), 26.85$ $\mathrm{m}$ a.s.l., 12.12.2013; 1 q (author's personal collection), Madras Christian College $\left(12.920297^{\circ} \mathrm{N}, 80.123583^{\circ} \mathrm{E}\right)$, Chennai, Tamil Nadu, 32 m a.s.1., 25.07.2013, John T.D. Caleb.

DESCRIPTION. MALE. Total length 8.85; carapace 3.52 long, 1.90 wide; abdomen 3.95 long, 1.62 wide. Width of eye row I 1.49; II 1.42; III 1.66. Eye size: AME 0.43, ALE 0.22, PME 0.05, PLE 0.24; ALE-PME 0.30, ALE-PLE 0.75. Chelicerae length
3.15. Sternum length 2.01. Leg spination. Femur I md $0-1$, pd 1; patella rv 1; tibia I pv 3, rv 4; metatarsus I pv 2, rv 2; tibia II pv 2-3, rv 0-3; metatarsus II pv 2, rv 2; femur III pd 1; tibia III pv $0-1$. Cephalic part dark brown, slightly higher than thoracic region; finely rugulose, covered with fine grey hairs. Thoracic region reddish, clearly separated with a distinct furrow, gradually sloping posteriorly (Figs 20, 21). Chelicerae shorter than carapace, brownish, rugulose, flattened dorsally chelicerae dentition with 10 promarginal teeth and 10 retromarginal teeth. Fang tapering gradually, slender with an apophysis (Fig. 22). Sternum brownish, slender, slightly overlapped by coxae I, II and III (Fig. 23). Legs I and II lighter, yellowish, with dark stripe on femur II. Coxa I orange, coxa II yellowish white. Leg III and IV brownish, trochanter IV and patella IV white (Figs 20,21, 23). Abdomen elongate, with dorsal scutum and a constriction; anterior region reddish brown, posterior region dark brown, covered with fine hairs uniformly (Fig. 20). Palps with rounded tegulum, with Cshaped seminal reservoir in the 2 to 3 o'clock position; embolus with two coils. RTA twisted, strongly curved, s-shaped, with developed flange (Figs 24, 25). 
FEMALE. Total length 8.01; carapace 3.10 long, 1.53 wide; abdomen 3.96 long, 1.76 wide. Width of eye row I 1.40; II 1.34; III 1.55. Eye size: AME 0.47, ALE 0.26, PME 0.05, PLE 0.24; ALE-PME 0.33, ALE-PLE 0.76. Sternum length 1.73. Leg spination. Tibia I pv 5, rv 5; metatarsus I pv 2, rv 2; tibia II pv 3, rv 3 ; metatarsus II pv 2, rv 2. Carapace coloration as in the male (Fig. 26). Chelicerae with 7 teeth on the prolateral margin and 8 teeth on the retrolateral margin (Fig. 28). Legs I and II lighter yellowish, except coxa I which is brownish. Legs III and IV brownish with white trochanter IV and patella IV. Thin dark brown stripes present on the lateral margins of first two pair of legs (Fig. 26). Abdomen elongate, devoid of scutum, with lateral white hairs near the weak constriction. Faint white transverse markings seen on the dorsum (Fig. 26). Epigyne with large, oval copulatory openings; copulatory ducts forming $\infty$-shaped twists before reaching spermathecae; lateral pockets present just above epigastric furrow (Figs 29, 30).

DISTRIBUTION. Pakistan to Indonesia [Edwards, Benjamin, 2009; WSC, 2016]; the records from India are shown in Map 1. 1869)

Myrmarachne plataleoides (O. Pickard-Cambridge,

Figs 31-54, Map 2.

Salticus plataleoides O. Pickard-Cambridge, 1869c: 68, pl. 6 , figs 61-65; Peckham, Peckham, 1892: 33, pl. 3, fig. 1.

Myrmarachne p.: Edmunds, Prószyński, 2003: 298, figs 1-7; Edwards, Benjamin, 2009: 16, figs 7; Benjamin, 2015: 38, figs 29A-D, 30A-D, 31A-E

Myrmaplata p.: Prószyński, 2016: 9, figs 1E-F, 2G, 3H.

MATERIAL. INDIA: $2 \sigma^{7}, 19$ (NCBS-AR125, AR053, AR057), 1 sub-adult 9 (NCBS-AR126), the NCBS campus $\left(13.071137^{\circ} \mathrm{N}, 77.580543^{\circ} \mathrm{E}\right)$, Bangalore, $896.92 \mathrm{~m}$ a.s.1., 18.05.2015, Rajesh Sanap \& Zeeshan Mirza.; $1 \sigma^{7}$ (NCBS-AR134), Madras Christian College $\left(12.919606^{\circ} \mathrm{N}, 80.122216^{\circ} \mathrm{E}\right)$, Chennai, Tamil Nadu, $32 \mathrm{~m}$ a.s.1., 25.03.2013, John Caleb T.D.; $1 \bigcirc^{7}$ (NCBSAR135), 1 sub-adult $\sigma^{\top}$ (NCBS-AR133), same college $\left(12.917013^{\circ} \mathrm{N}, 80.120572^{\circ} \mathrm{E}\right), 32 \mathrm{~m}$ a.s.1., 9.05.2013, John Caleb T.D. \& Nagoor Meerasa Mohammed.

DESCRIPTION. MALE (NCBS-AR125). Total length 7.76; carapace 3.26 long, 1.62 wide; abdomen 3.57 long, 1.45 wide. Width of eye row I 1.36; II 1.27; III 1.53. Eye size: AME 0.45, ALE 0.20, PME 0.10, PLE 0.22; ALE-PME 0.26, ALE-PLE 0.81. Chelicerae length 3.30. Sternum length 1.92. Leg spination. Femur I pr 2-3, di 1; tibia I pv 4, rv 4; metatarsus I pv 2, rv 2; femur II pr 1, md 1, pd 1; tibia II pv 1, rv 2; metatarsus II pv 2, rv 2; femur III pr 1-2, md 0-1, di 1; femur IV pr 1. Cephalic part flat dorsally, finely rugulose, covered with fine grey hairs. Thoracic region lower than cephalic region, convex dorsally, sloping posteriorly (Figs 31, 32). Chelicerae nearly as long as carapace, brownish with black tip near base of fang, basal half flattened dorsally, later half distended (Fig. 31 ); chelicerae dentition with 5 promarginal teeth and 10 retromarginal teeth. Fang long and slender without apophysis (Fig. 34). Sternum yellowish, slender, slightly overlapped by coxae II (Fig. 33). All leg segments orangish, except for white tarsus, trochanter IV and patella IV (Figs 31, 32). Abdomen elongate-oval, covered with fine grey hairs (Fig. 31). Palps with rounded tegulum, with C-shaped seminal reservoir in the 1 to 2 o'clock position; embolus with two coils. RTA short, thick and pointed tip with broad base (Figs 35, 36, 45, 46).

FEMALE. Total length 9.59; carapace 3.72 long, 1.72 wide; abdomen 4.59 long, 2.16 wide. Width of eye row I 1.45; II 1.38; III 1.69. Eye size: AME 0.50, ALE 0.21, PME 0.07, PLE 0.22; ALE-PME 0.36, ALE-PLE 0.92. Sternum length 2.04. Leg spination. Tibia I pv 4, rv 4; metatarsus I pv 2, rv 2; tibia II pv 1, rv 1; metatarsus II pv 2, rv 2. Coloration as in the male (Figs 49, 50). Chelicerae with 6 teeth on the prolateral margin and 10 teeth on the retrolateral margin (Fig. 52). Thin black stripes present on the lateral margins of first two pair of legs (Fig. 50). Epigyne with round copulatory openings; median pocket present just above epigastric furrow (Fig. 53).

VARIATION. DARK MALE (NCBS-AR134). Total length 6.03; carapace 2.76 long, 1.65 wide; abdomen 2.72 long, 1.54 wide. Width of eye row I 1.39; II 1.26; III 1.50. Eye size: AME 0.46, ALE 0.23, PME 0.06, PLE 0.22; ALE-PME 0.33, ALE-PLE 0.79. Chelicerae length 3.30. Sternum length 1.6. Leg spination. Femur I md 2, pd 1; tibia I pv 4-6, rv 4-6; metatarsus I pv 2, rv 2; femur II md 1, pd 1; tibia II pv 0-2, rv 2-3; metatarsus II pv 2, rv 2. Cephalic part flat dorsally, finely rugulose, covered with longer, whitish hairs. Thoracic region lower than cephalic region, slightly convex dorsally (Figs 37-39). Chelicerae longer than cephalothorax, black, swollen at apex, finely rugulose with furrows; chelicerae dentition with 6-7 promarginal teeth (5-6 distal, 1 proximal) and 10-15 retromarginal teeth. Fang blackish, long, slender without apophysis, slightly bent at the tip (Fig. 40). Sternum light brown, slender, slightly overlapped by coxae I, II and III (Fig. 41). Femur of all legs dark brown. Patella, tibia, metatarsus and tarsus lighter, yellowish in Leg I, II \& III. Trochanter IV white and patella IV whitish, with dark brown patch dorsally. Abdomen oval with slight constriction anteriorly; blackish, covered with dense whitish hairs (Figs 37, 38). Palps with small rounded tegulum, with seminal reservoir in the retrolateral position; embolus with two coils. RTA not twisted, broad at the base, pointed at the tip with moderately developed flange (Figs 42, 43, 47, 48).

REMARKS. Both the light and dark colour morphs of this species were collected from Chennai and Bangalore (Figs 31, 37, 44, 54). Benjamin [2016; pers. comm.] argued that the dark specimens from Chennai should be treated as $M$. plataleoides, since dark colour morphs are common in this species. However, the specimens from Chennai also show a morphological variation and are likely to be younger, yet to undergo more moults before finally getting their characteristic elongated form. The mature forms have the carapace with the markedly constricted lateral margins between legs II and III (Figs 31, 33) and the sternum margins being 

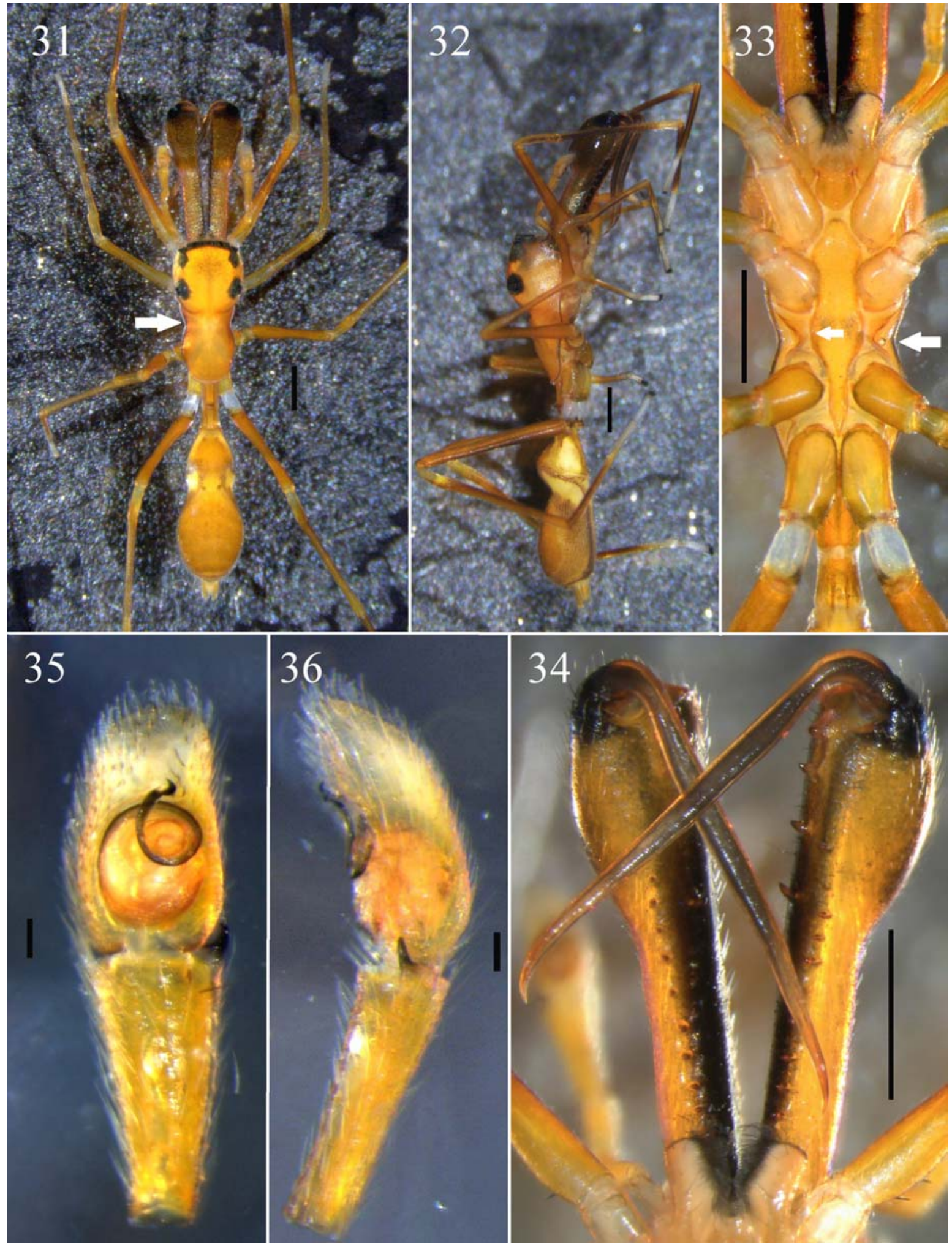

Figs 31-36. The male of Myrmarachne plataleoides (O. Pickard-Cambridge, 1869) from Bangalore. 31 — body, dorsal view; 32 ditto, lateral view; 33 - maxillae, labium, sternum and coxae, ventral view; 34 - chelicerae, ventral view; 35 - palp, ventral view; 36 ditto, retrolateral view. Scale bars: $1 \mathrm{~mm}(31-44), 0.1 \mathrm{~mm}(35,36)$.

Рис. 31-36. Самец Myrmarachne plataleoides (O. Pickard-Cambridge, 1869) из Бангалура. 31 - тело, вид сверху; 32 - тоже, вид сбоку; 33 - максилла, лабиум, стернум и вертлуги, вид снизу; 34 - хелицеры, вид снизу; 35 — пальпа, вид снизу; 36 - тоже, вид сбоку-сзади. Масштаб: 1 мм (31-44), 0,1 мм $(35,36)$. 

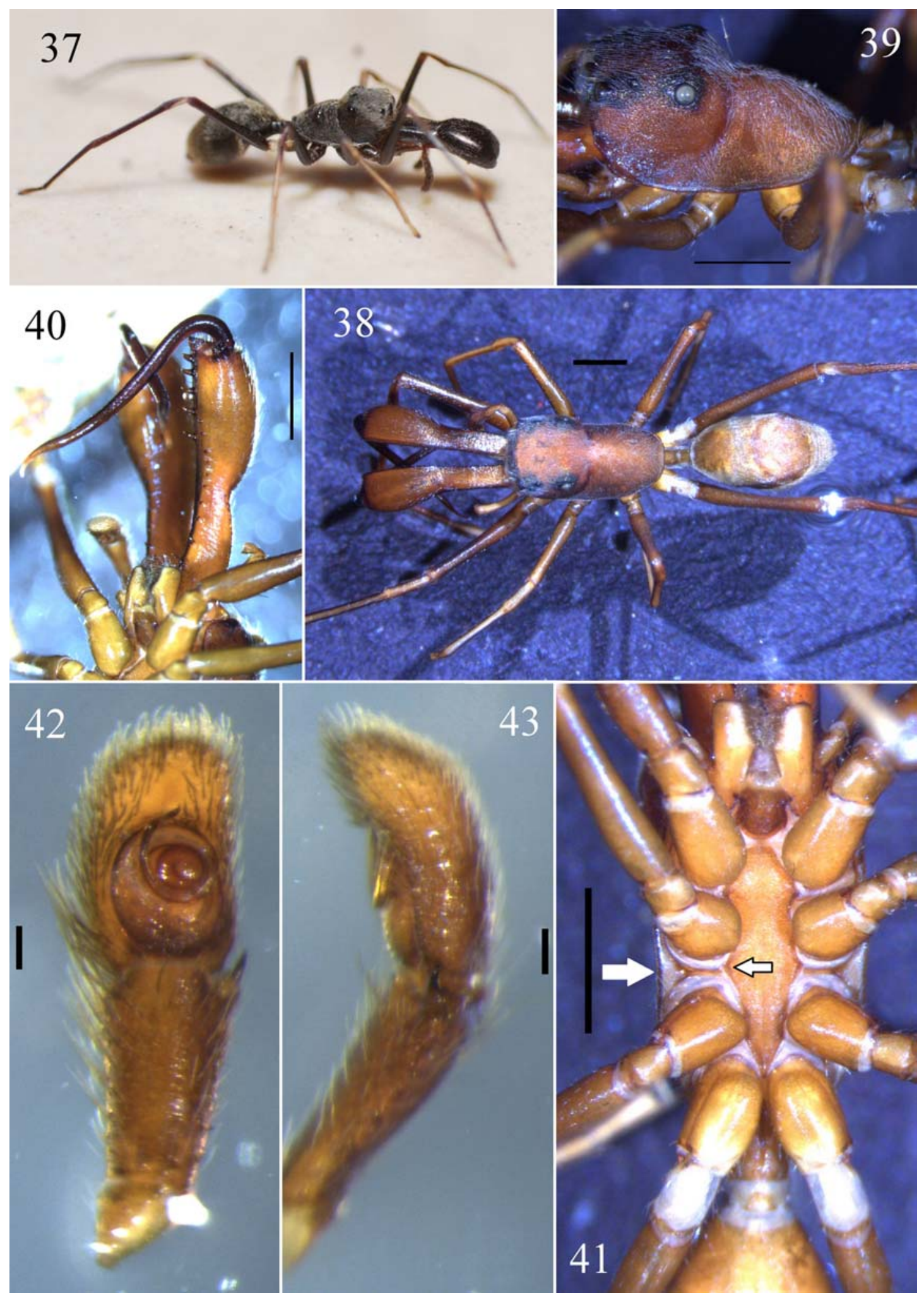

Figs 37-43. The male of Myrmarachne plataleoides (O. Pickard-Cambridge, 1869) from Chennai. 37 — body, lateral view; 38 ditto, dorsal view; 39 - carapace, lateral view; 40 - chelicerae, ventral view; 41 - maxillae, labium, sternum and coxae; 42 - palp, ventral view; 43 - ditto, retrolateral view. Scale bars: $1 \mathrm{~mm}(38-41), 0.1 \mathrm{~mm}(42,43)$.

Рис. 37-43. Самец Myrmarachne plataleoides (O. Pickard-Cambridge, 1869) из Хенная. 37 — тело, вид сбоку; 38 — тоже, вид сверху; 39 - головогрудь, вид сбоку; 40 - хелицеры, вид снизу; 41 - максилла, лабиум, стернум и вертлуги; 42 - пальпа, вид снизу; 43 - тоже, вид сбоку-сзади. Масштаб: 1 мм (38-41), 0,1 мм $(42,43)$. 

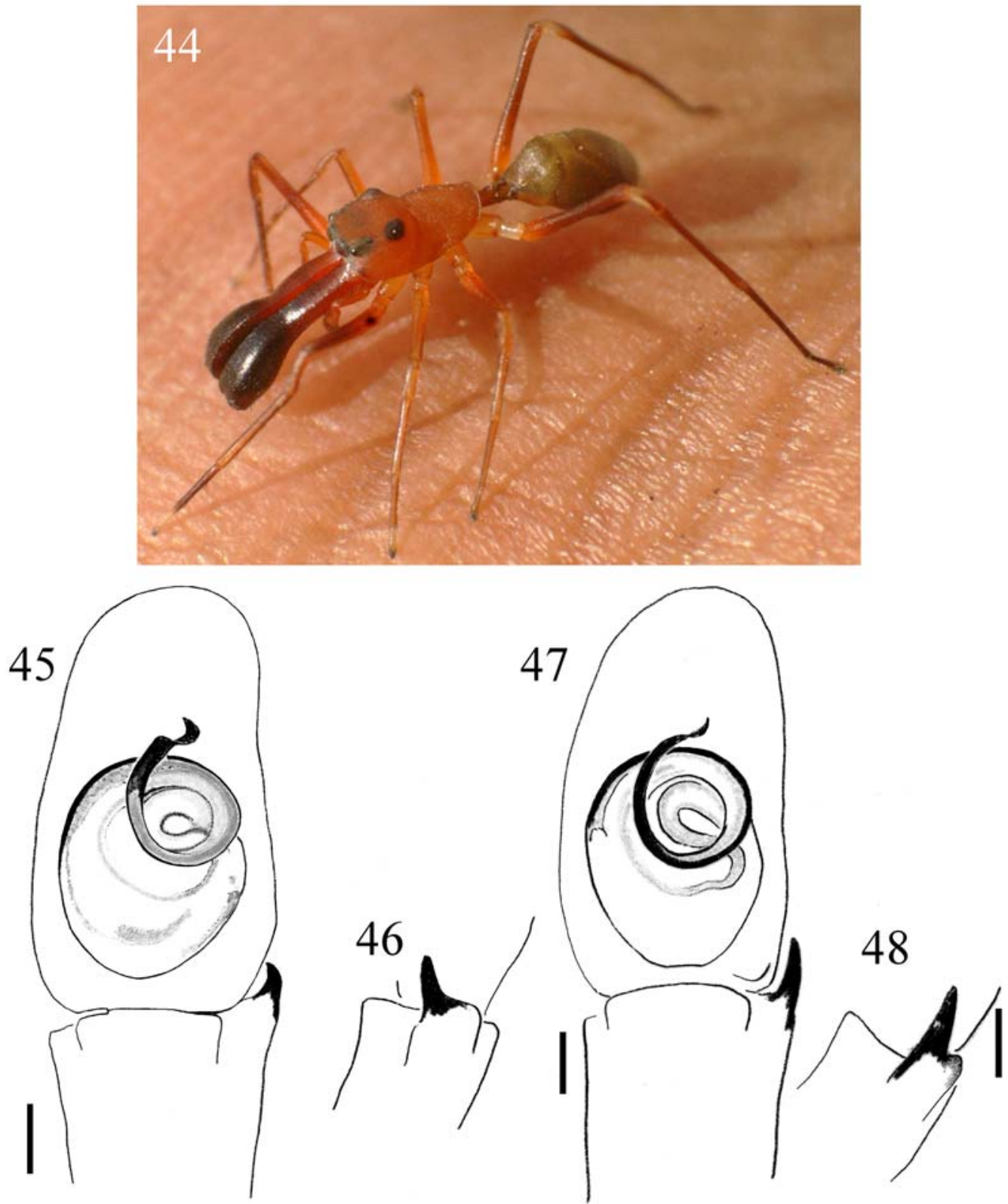

Figs 44-48. The male of Myrmarachne plataleoides (O. Pickard-Cambridge, 1869): 44 — body dorso-lateral view; 45 — palp, ventral view; 46 - ditto, retrolateral view showing RTA; 47 - ditto, ventral view; 48 - ditto, retrolateral view showing RTA. Scale bars: $0.1 \mathrm{~mm}$ (45-48).

Рис. 44-48. Самец Myrmarachne plataleoides (O. Pickard-Cambridge, 1869): 44 - тело, вид сверху-сбоку; 45 - пальпа, вид снизу; 46 - тоже, вид сбоку-сзади, показывающий RTA; 47 - тоже, вид снизу; 48 - тоже, вид сбоку-сзади, показывающий RTA. Масштаб: 0,1 мм (45-48).

sub-parallel (cf. Figs 33 and 41); the size of the pedicel is longer (cf. Figs 31 and 38); the abdomen has a deeper constriction between the anterior and posterior scuta (cf. Fig. 31 with Figs 38 and 44).

It should be mentioned that Prószyński [2016b] erected a separate genus Myrmaplata Prószyński, 2016 for this species.

DISTRIBUTION. India (Map 2), Sri Lanka, China, Southeast Asia [Benjamin, 2015; WSC, 2016].

\section{Myrmarachne prava (Karsch, 1880)}

Figs 55-65, Map 2.

Salticus pravus Karsch, 1880: 395.

Myrmarachne prava: Benjamin, 2015: 26, figs 23A-E, 24AD, 25A-D, 26A-D, 27A-C, 28A-E.

MATERIAL. INDIA: 1 † (NCBS-AR138), Kadapa $\left(14.451506^{\circ} \mathrm{N}, 78.794684^{\circ} \mathrm{E}\right), 138 \mathrm{~m}$ a.s.1., 14.01.2013, John T.D. Caleb; 1 ㅇ (NCBS-AR139), Thirumullaivoyal $\left(13.125267^{\circ} \mathrm{N}\right.$, $\left.80.135626^{\circ} \mathrm{E}\right), 26.85 \mathrm{~m}$ a.s.1., 24.01.2014, John T.D. Caleb. 

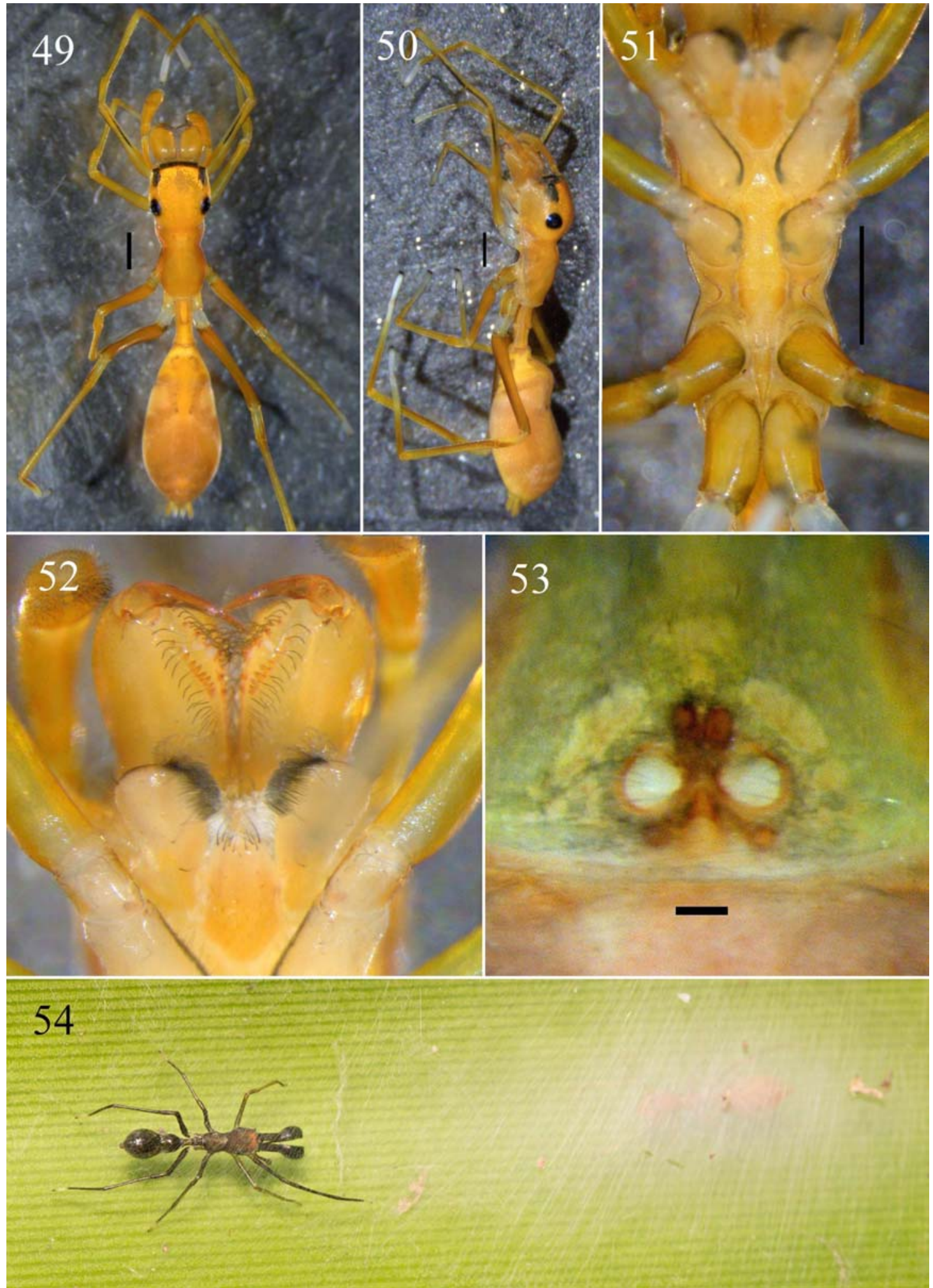

Figs 49-54. The female of Myrmarachne plataleoides (O. Pickard-Cambridge, 1869): 49 — body, dorsal view; 50 — ditto, lateral view; 51 - sternum and coxae; 52 - chelicerae, ventral view; 53 - epigyne, ventral view; 54 - dark male guarding or courting a female inside the silken retreat. Scale bars: $1 \mathrm{~mm}(49-51), 0.1 \mathrm{~mm}$ (53). Figs 53, 54 were kindly provided by Rajesh Sanap.

Рис. 49-54. Самка Myrmarachne plataleoides (O. Pickard-Cambridge, 1869): 49 — тело, вид сверху; 50 — тоже, вид сбоку; 51 стернум и вертлуги; 52 - хелицеры, вид снизу; 53 - эпигина, вид снизу; 54 - темный самец охраняющий самку внутри паутинового убежища, либо ухаживающий за ней. Масштаб: 1 мм (49-51), 0,1 мм (53). Рис. 53, 54 любезно предосталены Раджешем Санапом. 


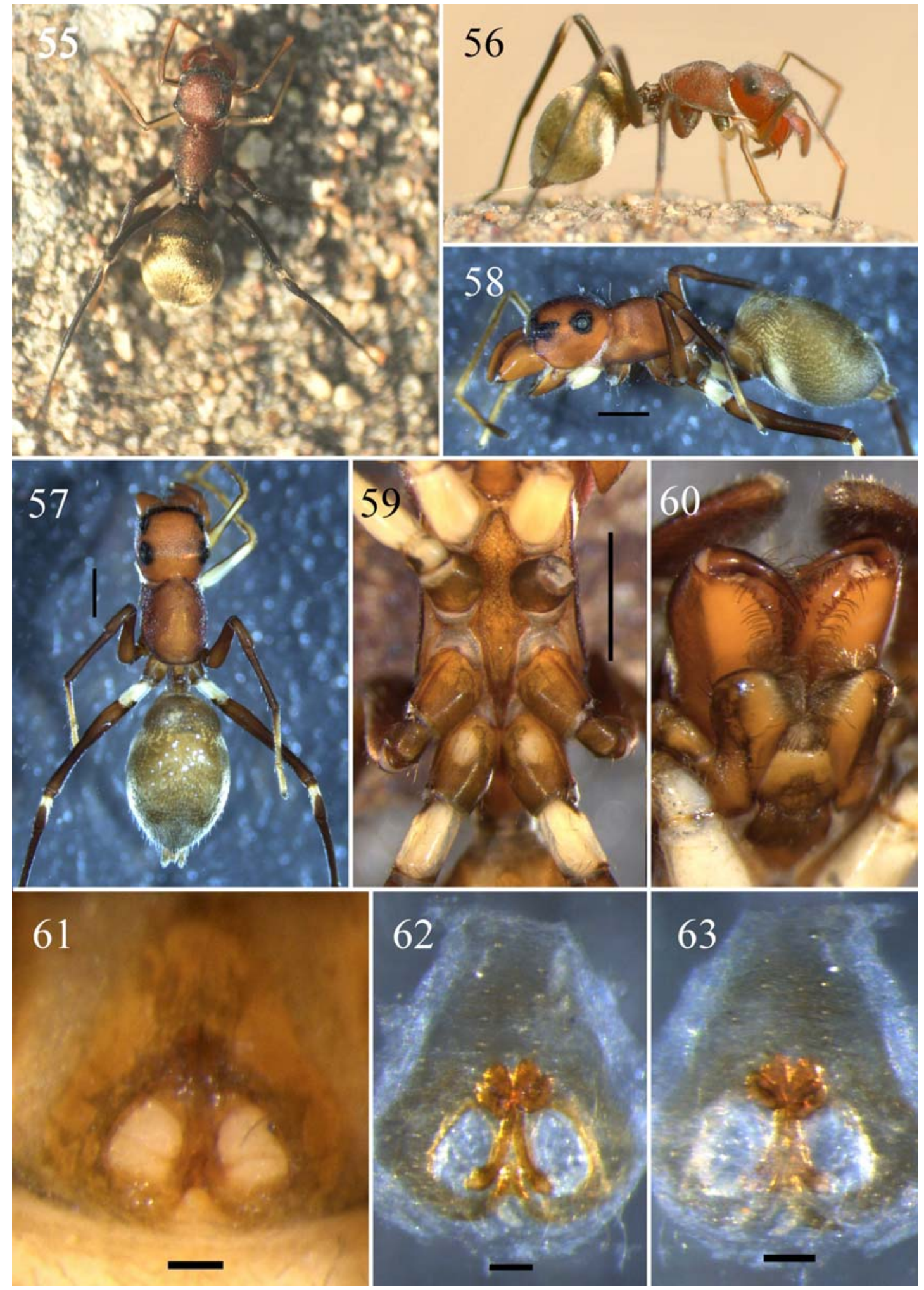

Figs 55-63. The female of Myrmarachne prava (Karsch, 1880). 55, 57 - body, dorsal view; 56, 58 - ditto, lateral view; 59 sternum and coxae; 60 - chelicerae, fangs, labium and maxillae; 61 - epigyne, ventral view; 62 - ditto, ventral view after clearing; 63 - spermathecae, dorsal view. Scale bars: $1 \mathrm{~mm}(57-60), 0.1 \mathrm{~mm}(61-63)$.

Рис. 55-63. Самка Myrmarachne prava (Karsch, 1880). 55, 57 - тело, вид сверху; 56, 58 - тоже, вид сбоку; 59 - стернум и вертлуги; 60 - хелицеры, максиллы и лабиум; 61 - эпигина, вид снизу; 62 - тоже, вид сверху после очистки; 63 - сперматека, вид сверху. Масштаб: 1 мм (57-60), 0,1 мм (61-63). 

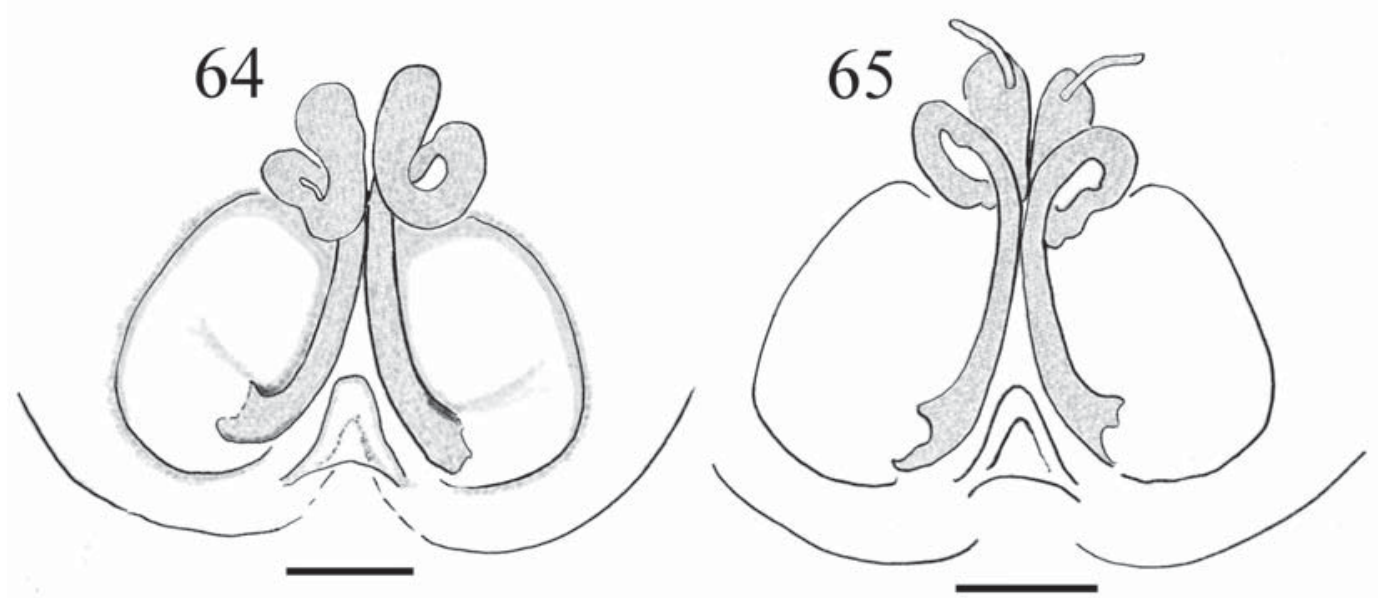

Figs 64-65. Copulatory organs of Myrmarachne prava (Karsch, 1880). 64 - spermathecae, ventral view; 65 - ditto, ventral view. Scale bars: $0.1 \mathrm{~mm}(64-65)$.

Рис. 64-65. Копулятивные органы Myrmarachne prava (Karsch, 1880). 64 - сперматека, вид сверху; 65 - тоже, вид снизу. Масштаб: 0,1 мм (64-65).

DESCRIPTION. FEMALE. Total length 7.05; carapace 3.15 long, 1.36 wide; abdomen 3.31 long, 2.10 wide. Width of eye row I 1.34; II 1.28; III 1.50. Eye size: AME 0.42, ALE 0.19, PME 0.05, PLE 0.22; ALE-PME 0.28, ALE-PLE 0.71. Sternum length 1.70. Leg spination. Tibia I pv 4, rv 4; metatarsus I pv 2, rv 2; tibia II pv 3, rv 2-3; metatarsus II pv 2, rv 2. Cephalic part reddish, flat dorsally, finely rugulose, covered with fine grey hairs. Thoracic region clearly separated with deep furrow, slightly lower than cephalic region, convex dorsally, sloping posteriorly (Figs 55-58). Chelicerae reddish, with 8 promarginal teeth and 7 retromarginal teeth. (Fig. 60). Sternum brownish, slender, slightly overlapped by coxae II (Fig. 59). Leg I and II yellowish with dark stripes laterally. Leg III and IV brownish with white trochanter IV and lighter patella IV, coxa I yellowish, coxa II-IV brownish. Abdomen oval, covered with fine yellowish brown hairs (Figs 55-58). Epigyne with large, oval copulatory openings; sclerotized copulatory ducts thinner, making a simple twist; median pocket present just above epigastric furrow (Figs 61-65).

DISTRIBUTION. India (Map 2), Sri Lanka [Benjamin, 2015; WSC, 2016].

Myrmarachne ramunni Narayan, 1915 Figs 66-75, Map 2.

Myrmarachne ramunni Narayan, 1915: 400-402, figs 4a-c; Benjamin, 2015: 43, figs 2E-F, 3E-F.

MATERIAL. INDIA: $2 \sigma^{7} \sigma^{7}$ (NCBS-AR136, AR137), Thirumullaivoyal $\left(13.125090^{\circ} \mathrm{N}, 80.135712^{\circ} \mathrm{E}\right), 26.85 \mathrm{~m}$ a.s.1., 1.04 .2013 , John T.D. Caleb.

DESCRIPTION. MALE. Total length 5.59; carapace 2.81 long, 1.62 wide; abdomen 2.47 long, 1.55 wide. Width of eye row I 1.34; II 1.27; III 1.43. Eye size: AME 0.46, ALE 0.21, PME 0.05, PLE 0.24; ALE-PME 0.27, ALE-PLE 0.65. Chelicerae length
2.98. Sternum length 1.57. Leg spination. Femur I md 1 , patella v 1; tibia I pv 5, rv 5; metatarsus I pv 2, rv 2; femur II md 1; tibia II pv 3, rv 3; metatarsus II pv 2, rv 2; femur III md 1. Cephalic part flat dorsally, covered with grey hairs (Fig. 66). Thoracic region separated with deep furrow, lower than cephalic region, convex dorsally, gradually sloping posteriorly (Fig. 69). Chelicerae blackish-brown, finely rugulose, long with narrow basal region and flattened apically into spatula shape (Fig. 67); chelicerae dentition with 10-13 promargin teeth and 8 retromargin teeth (Fig. 68). Fang rugulose, long with a proximal arch and an apophysis (Fig. 70). Sternum dark, slender, strongly overlapped by coxae I, II \& III (Fig. 71). Legs yellowish brown, lateral stripes slightly diffused with the light color on legs I \& II. Coxa I yellowish, coxa II, III brownish, coxa IV yellowish-brown. Leg IV with dark femur, metatarsus, tibia and tarsus. Trochanter IV white, patella IV white with dark dorsal distal region. Abdomen oval, covered with fine hairs (Fig. 66). Palps with rounded tegulum, with $\mathrm{C}$-shaped seminal reservoir from the 1 to 2 o'clock position; embolus with two coils. RTA thick, broad at the base, pointed at the tip, moderately developed flange (Figs 72-75).

REMARKS. M. ramunni was originally described from Madras (now Chennai). The material treated here is also collected from the outskirts of Chennai City. The male chelicerae are distinct and useful in identifying this species as mentioned by Benjamin [2015] (cf. figs 67 and 70 with figs 4a-b in Narayan [1915] and 2E in Benjamin [2015]).

DISTRIBUTION. India (Map 2), Pakistan and Sri Lanka [Benjamin, 2015; WSC, 2016].

ACKNOWLEDGEMENTS. I am grateful to Prof Dr Jerzy Prószyński (Warsaw, Poland) for the fruitful discussion on the salticid fauna of the Indian subcontinent. I am 


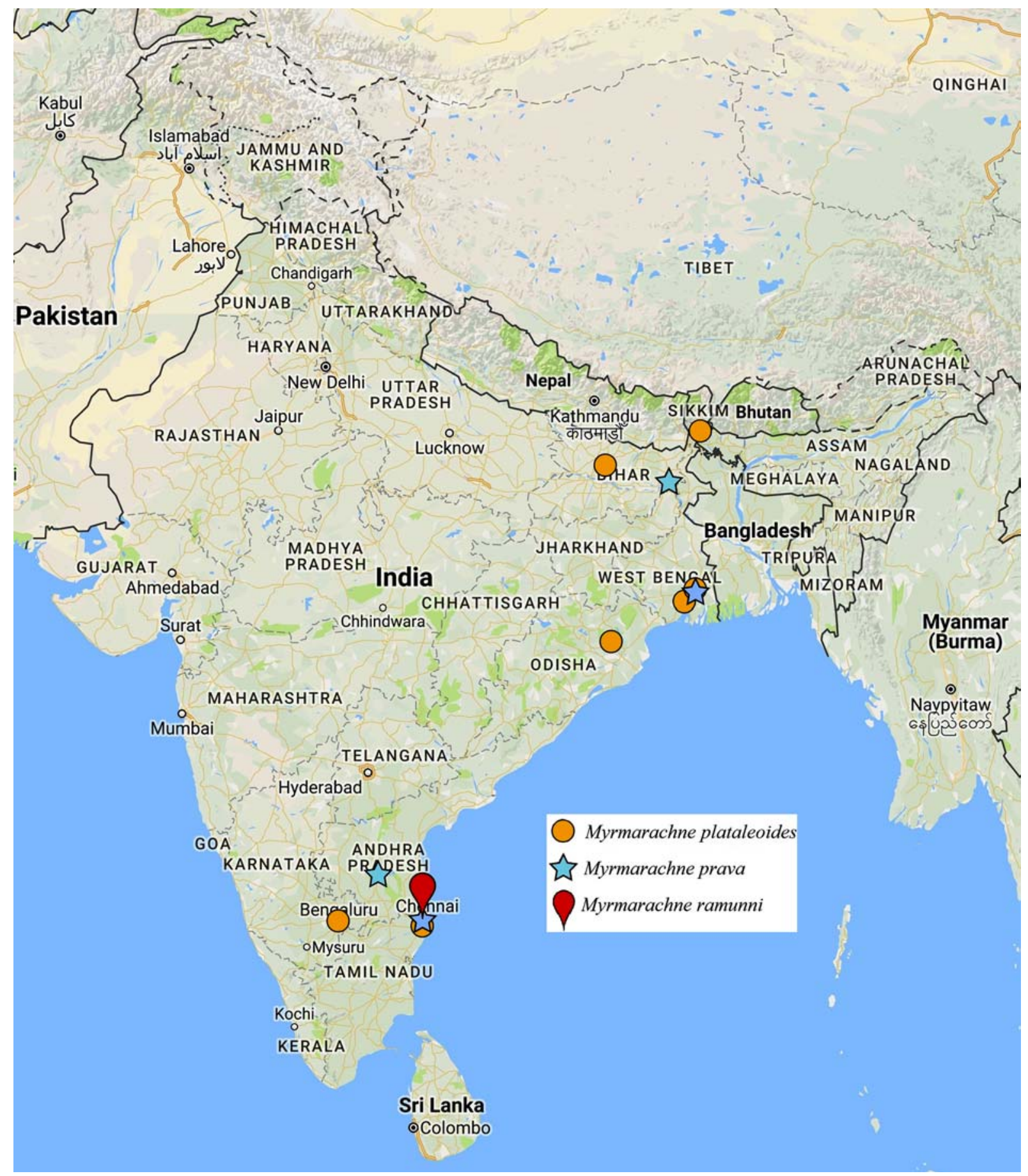

Map 2. Distributional records of Myrmarachne spp. in India.

Карта 2. Точки находок Myrmarachne spp. в Индии.

thankful to Dr Krushnamegh Kunte, Lab 8, National Centre for Biological Sciences, (Bangalore, India) for allowing me to use the stereo microscope facility and to deposit the studied specimens. Thanks go to Nagoor Meerasa Mohammed and Barnaba Thomas for their kind assistance during the field trips. Special thanks go to Rajesh Sanap and Zeeshan Mirza for collecting specimens and for providing me with the photos of specimens from the NCBS campus,
Bangalore. I wish also to thank the Zoology Department of the Madras Christian College for providing a digital camera for photographic documentation. Special thanks to Dr Dmitri Logunov (Manchester, UK) for his editorial and linguistic help which has improved the ms greatly. Finally, I wish to thank my parents David and Udaya Prasanna for their continual encouragement and financial support throughout my research. 

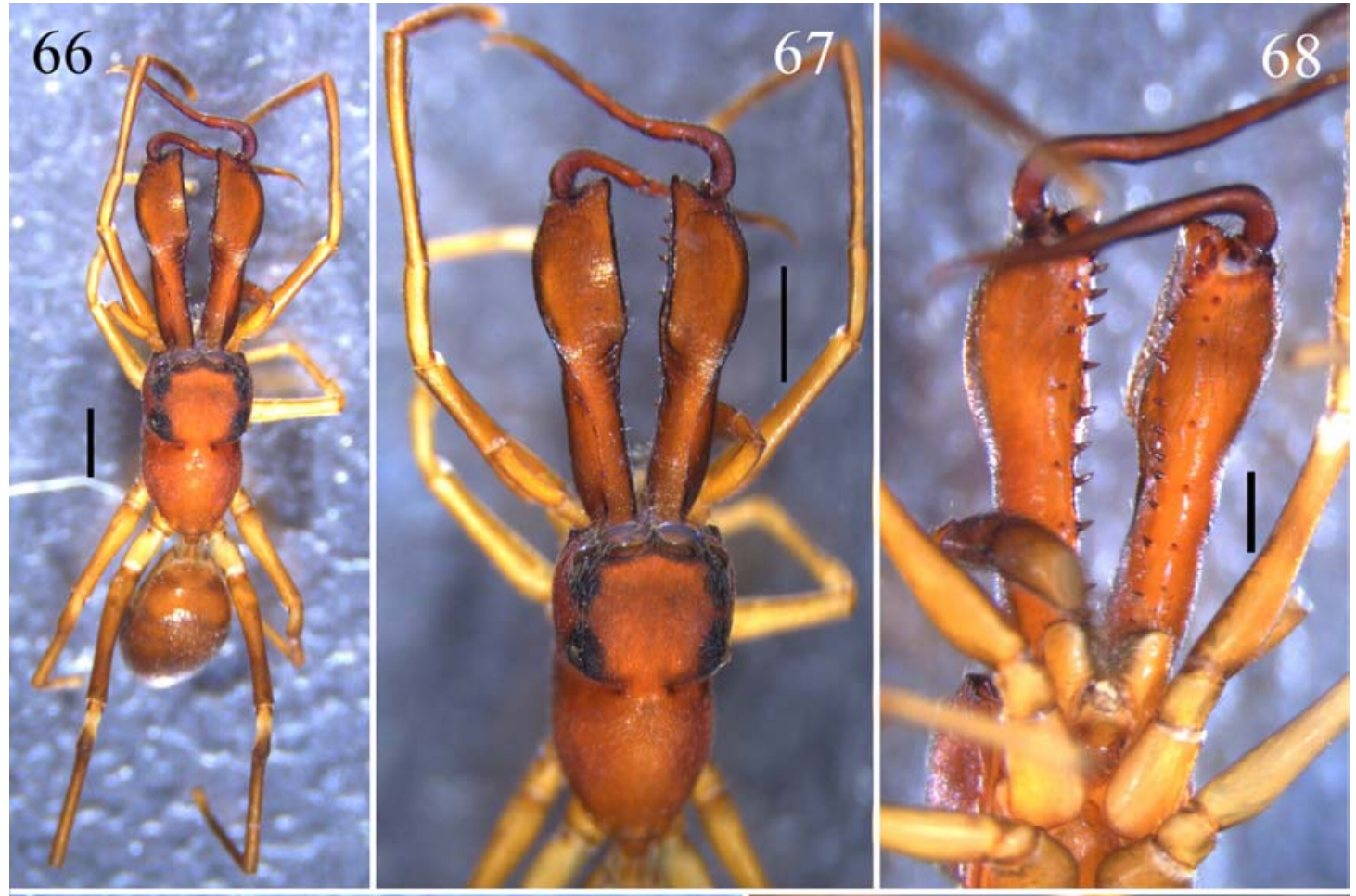
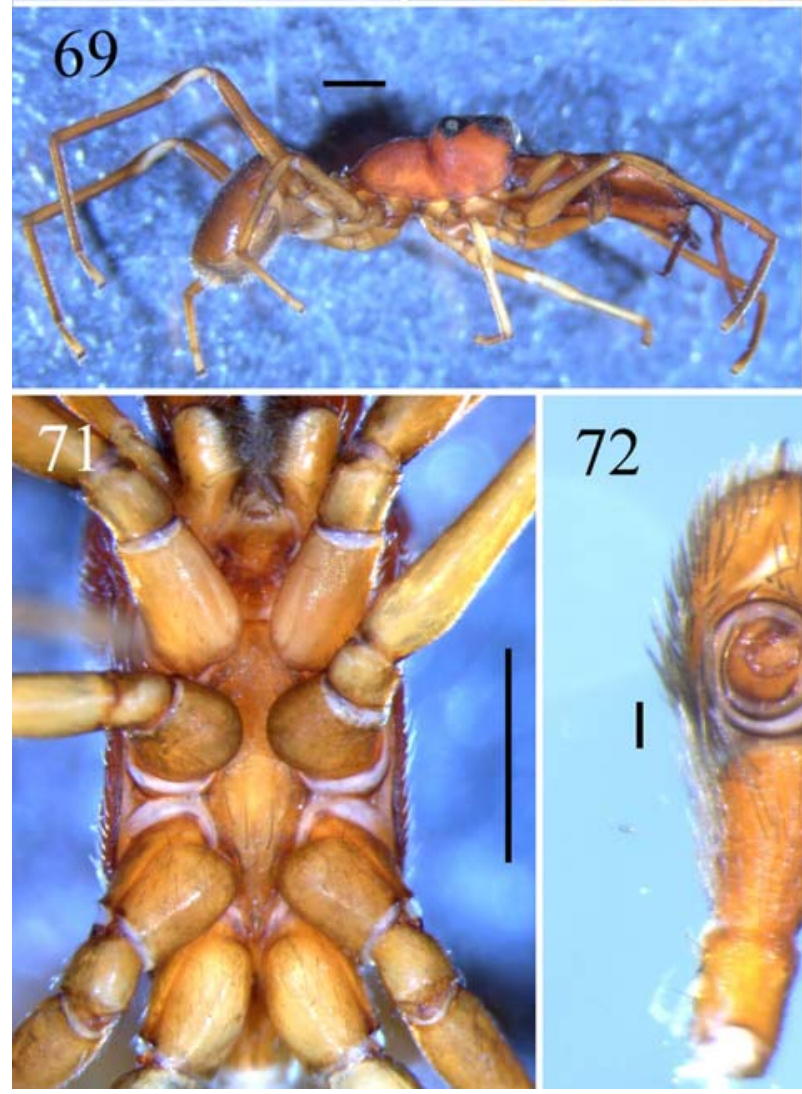
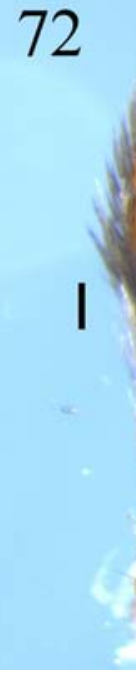

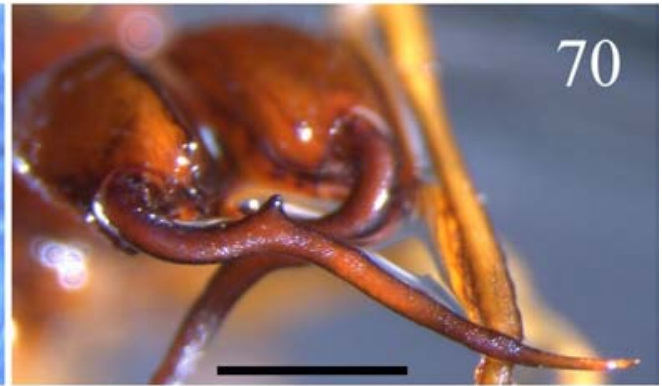

73

Figs 66-73. The male of Myrmarachne ramunni Narayan, 1915. 66 - body, dorsal view; 67 - chelicerae and carapace, dorsal view; 68 - chelicerae, ventral view; 69 - body, lateral view; 70 - cheliceral fang, anterior view; 71 - maxillae, labium, sternum and coxae; 72 - palp, ventral view; 73 - ditto, retrolateral view. Scale bars: $1 \mathrm{~mm}$ (66-67, 69-71), $0.5 \mathrm{~mm}(68), 0.1 \mathrm{~mm}(72-73)$.

Рис. 66-73. Самец Myrmarachne ramunni Narayan, 1915. 66 - тело, вид сверху; 67 - хелицеры и головогрудь, вид сверху; 68 - хелицеры, вид снизу; 69 - тело, вид сбоку; 70 - коготь хелицеры, вид спереди; 71 - максилла, лабиум, стернум и вертлуги; 72 - пальпа, вид снизу; 73 - тоже, вид сбоку-снизу. Масштаб: 1 мм (66-67, 69-71), 0,5 мм (68), 0,1 мм (72-73). 


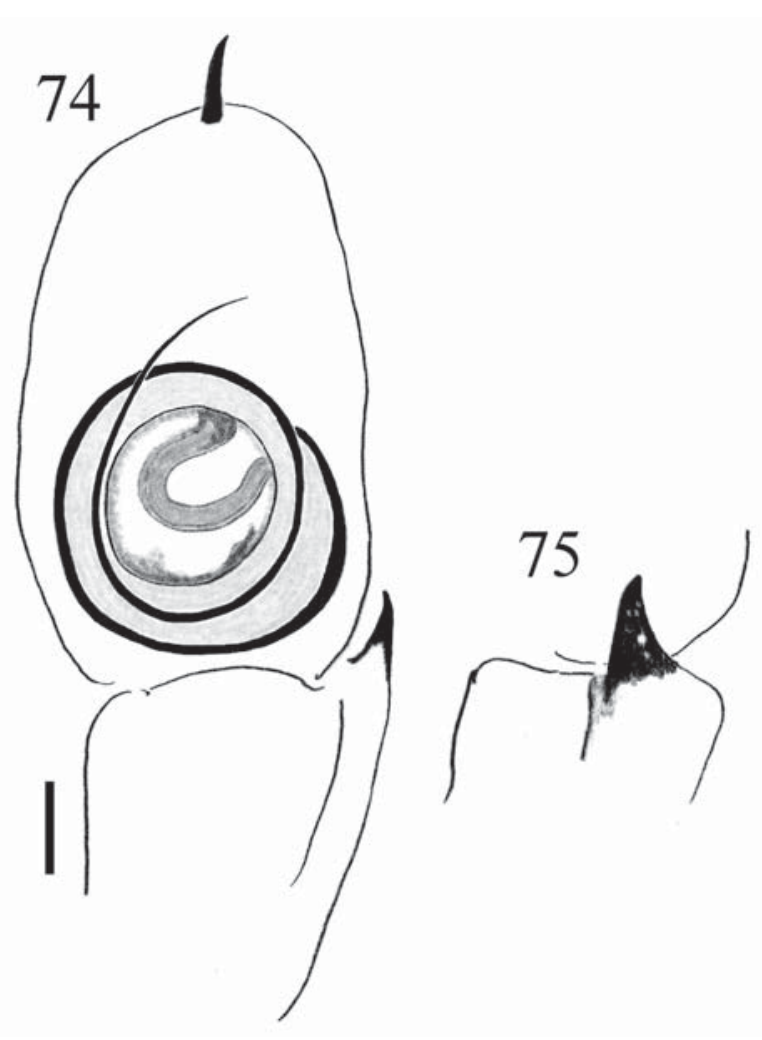

Figs 74-75. Copulatory organs of Myrmarachne ramunni Narayan, 1915. 74 - palp, ventral view; $75-$ ditto, retrolateral view showing RTA. Scale bars: $0.1 \mathrm{~mm}$ (74-75).

Рис. 74-75. Копулятивные органы Myrmarachne ramunni Narayan, 1915. 74 - пальпа, вид снизу; 75 - тоже, вид сбокусзади, показывающий RTA. Масштаб: 0,1 мм (74-75).

\section{References}

Benjamin S.P. 2015. Model mimics: antlike jumping spiders of the genus Myrmarachne from Sri Lanka // J. Nat. Hist. Vol.49. No.43-44. P.2609-2666.

Edmunds M., Prószyński J. 2003. On a collection of Myrmarachne spiders (Araneae: Salticidae) from peninsular Malaya // Bull. Br. Arachnol. Soc. Vol.12. Pt.7. P.297-323.

Edwards G.B., Benjamin S.P. 2009. A first look at the phylogeny of the Myrmarachninae, with rediscovery and redescription of the type species of Myrmarachne (Araneae: Salticidae) // Zootaxa. Vol.2309. P.1-29.

Prószyński J. 1992. Salticidae (Araneae) of India in the collection of the Hungarian National Natural History Museum in Budapest // Ann. Zool., PAN. T1.44. No.9. P.165-277.

Prószyński J. 2016a. Salticidae (Araneae) of the World, Chapter II; online at: http://www.peckhamia.com/salticidae (accessed on 12th April 2016)

Prószyński J. 2016b. Delimitation and description of 19 new genera, a subgenus and a species of Salticidae (Araneae) of the world // Ecol. Montenegrina. Vol.7. P.4-32.

Wanless F.R. 1978. A revision of the spider genera Belippo and Myrmarachne (Araneae: Salticidae) in the Ethiopian region // Bull. Br. Mus. Nat. Hist. (Zool.). Vol.33. No.1. P.1-139.

WSC. 2016. World Spider Catalog. Natural History Museum Bern; online at: http://wsc.nmbe.ch,version 17.5 (accessed on 2 nd August 2016).

Yaginuma T. 1967. Revision and new addition to fauna of Japanese spiders, with descriptions of seven new species // Literary Department Review, Otemon Gakuin University, Osaka Vol.1. P.87-107.

Yamasaki T., Ahmad A.H. 2013. Taxonomic study of the genus Myrmarachne of Borneo (Araneae: Salticidae) // Zootaxa. Vol.3710. No.6. P.501-556.

Yamasaki T., Edwards G.B. 2013. The genus Myrmarachne (Araneae, Salticidae) in Flores, Indonesia // ZooKeys. Vol.299. P.1-20.

Responsible editor D.V. Logunov 